\title{
Changing Communication Path to Maintain Connectivity of Mobile Robots in Multi-Robot System using Multistage Relay Networks
}

\author{
Ryo Odake, Kei Sawai \\ Graduate School of Engineering \\ Toyama Prefectural University, Toyama, Japan
}

\begin{abstract}
Mobile robots are being increasingly used to gather information from disaster sites and prevent further damage in disaster areas. Previous studies discussed a multi-robot system that uses a multistage relay backbone network to gather information in a closed space after a disaster. In this system, the mobile robot explores its search range by switching the connected nodes. Here it is necessary to maintain the communication quality required for the teleoperation of the mobile robot and to send and receive packets between the operator PC and the mobile robot. However, the mobile robot can become isolated when it is not able to maintain the communication quality required for teleoperations in the communication path after changing the nodes. This paper proposes a method to change the communication path of a mobile robot while maintaining its communication connectivity. In the proposed method, the mobile robot changes its route while maintaining communication connectivity without any communication loss time by connecting to two nodes.
\end{abstract}

Keywords-Multi-robot; multistage relay network; communication connectivity; changing communication path

\section{INTRODUCTION}

After a disaster occurs, disaster reduction activities are performed in the affected area to prevent the damage from spreading. In the implementation of disaster reduction activities, information needs to be gathered to determine the damage status [1-2]. Existing infrastructure, such as surveillance cameras, drone aerial photographs, and rescue teams, can be used to gather such information [3-6]. However, in some cases, the existing infrastructure cannot be used because of infrastructure malfunction or lack of power supply. Also, it is difficult for people to control the drone based on the camera images. Therefore, the use of drones is not effective in enclosed spaces after a disaster. Moreover, there is a risk of endangering human lives or inducing secondary disasters during information gathering by rescue teams. Therefore, the use of mobile robots is widely preferred for gathering information in enclosed spaces after disasters [7-11].

Two communication methods are adopted for mobile robots: wired and wireless. Wired communication helps maintain a stable communication quality and power supply to the mobile robot by using cables [12]. However, cables can get disconnected and communication with the mobile robots can be interrupted when cables become tangled with obstacles or the wheels of the mobile robot. Wireless communication has a high runnability because of the absence of physical restrictions using cables [13-14]. However, in wireless communication, mobile robots may become isolated when radio waves are hindered by obstacles. Therefore, in an enclosed space after a disaster, it is necessary to use the communication method that best matches the purpose and the situation of the disaster area [15-16]. This paper discusses a method for gathering information using wireless communication in environments where it is difficult to explore with a mobile robot using wired communication.

Robot wireless sensor networks (RWSNs) involve the teleoperation of mobile robots using wireless communication [17-19]. In an RWSN system, a mobile robot expands its search range by deploying a relay node called a sensor node (SN) in its path (Fig. 1). Therefore, the RWSN can gather information without depending on the existing infrastructure. In a network that uses multistage relaying, such as RWSN, the communication quality decreases as the number of relays and the distance between the nodes increases. Therefore, it is difficult to maintain communication connectivity of mobile robots in a multistage relay network; these networks are mainly operated by single robots. However, there is a limit to the range searched by a single robot in a large-scale facility. Therefore, this study discusses a multi-robot system that uses a multistage relay backbone network (Fig. 2).

In the proposed multi-robot system, a static multistage relay network is constructed by deploying SNs equipped with a single mobile robot first. Then, this system connects the mobile robot to the constructed network, and the mobile robot explores the search range by switching its connection between the nodes. Then, the mobile robot exhaustively explores within the network construction range while changing the search range by switching between the nodes. However, when changing the node to be connected, the operator experiences communication loss time with the mobile robot and cannot obtain environmental information from the mobile robot. Moreover, there is the risk of isolation when a mobile robot fails to connect to a node or is unable to reconnect. In addition, the operator cannot determine whether the communication quality required for the teleoperation of the mobile robot can be maintained in the communication path after changing the node to which the robot needs to connect. Therefore, there is a risk that the mobile robot becomes isolated without maintaining the communication quality. 


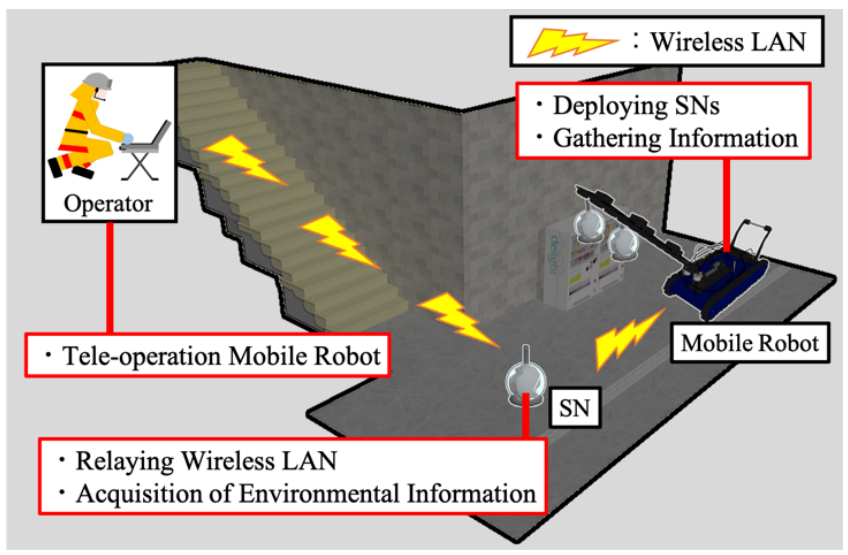

Fig. 1. Gathering Information by Mobile Robot (Robot Wireless Sensor Networks : RWSN).

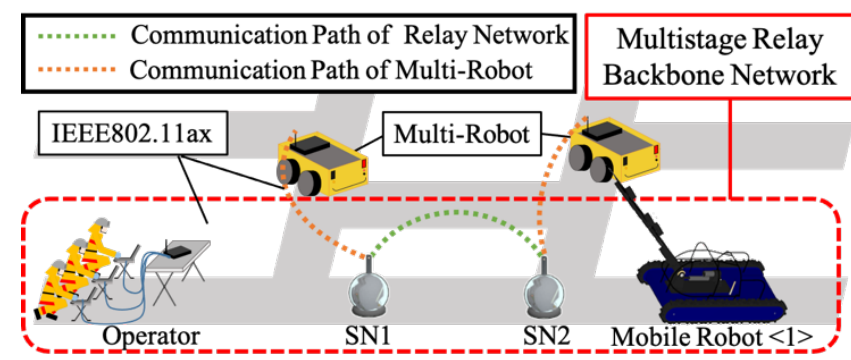

Fig. 2. Multi-Robot System using Multistage Relay Network.

To solve these problems, this paper proposes a method to change the communication path so that the communication connectivity of mobile robots is maintained. In the proposed method, the mobile robot teleoperates with two communication paths to eliminate any communication loss with the mobile robot. The communication quality required for the teleoperation of the mobile robot in the communication path is maintained after changing the nodes to be connected to; this method obtains the communication quality of the changed communication path before changing the communication path. The experiment in this paper confirmed that the required communication quality can be maintained in the communication path after changing the connected node using the proposed method. And the experiment showed the effectiveness of the proposed method.

\section{Multi-Robot System using Multistage Relay NETWORK}

\section{A. Multi-robot Information Gathering}

Many wireless teleoperation systems for mobile robots are based on the transmission control protocol/Internet protocol (TCP/IP). TCP/IP is highly compatible with mobile robot communication because most control systems of mobile robots use PCs. Therefore, socket communication is often adopted for mobile robot communication, and information communication is typically done by packet transmission and reception. Therefore, RWSN adopted the wireless LAN as the communication method. The operator receives packets containing information about the camera and sensor, which is acquired by the mobile robot, and this information is used to teleoperate the mobile robot (Fig. 3).

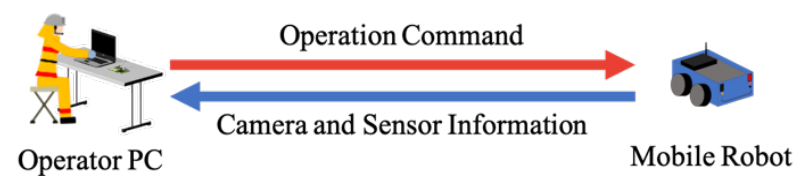

Fig. 3. Teleoperating Mobile Robot.

In previous research, there are systems that distribute and explore a large number of robots in the environment [20-21]. However, this system dynamically changes the route to send the information to the operator, which results in a misalignment of the reception intervals of the operation command packets. In addition, it is difficult to send large amounts of data, such as videos, using this system. These problems limit the operability and the ability of the mobile robot to gather information. Therefore, in this paper, the mobile robot is explored by connecting it to a static multistage relay network, such as the RWSN.

\section{B. Flow and Requirements for Information Gathering by Multi-robot System using Multistage Relay Network}

The process of the multi-robot operation using the multistage relay network is shown below (Fig. 4).

- Construction range of a multistage relay backbone network is expanded with the mobile robot $<1>$ such as RWSN.

- Multiple mobile robots are connected to the constructed backbone network.

- Mobile robot $<1>$ and the other mobile robots search within the network construction range while switching the nodes to be connected.

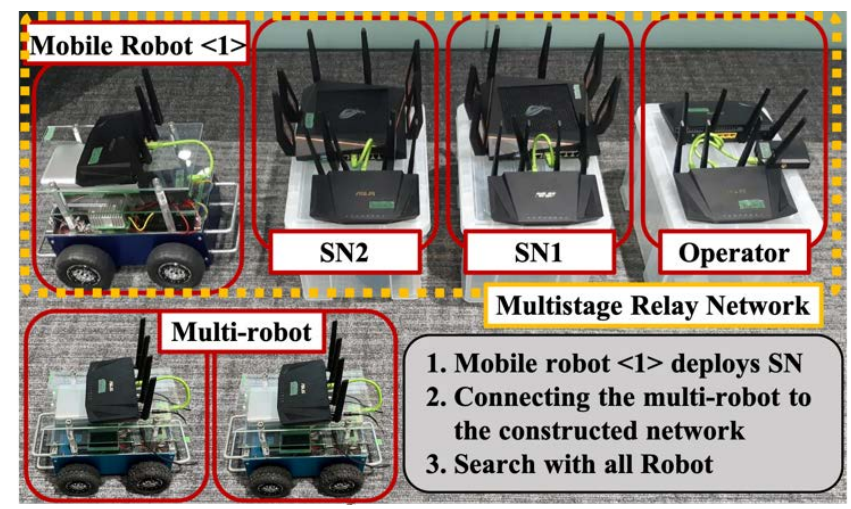

Fig. 4. Device of each Node that Constitutes Multi-robot System.

Based on the results of the operations of the robots at the damaged nuclear power plant, this study defined the network requirements of the multi-robot system as below.

- Teleoperation of a total of three or more mobile robots.

- Maintaining a throughput of $20 \mathrm{Mbps}$ or higher in the communication path between each operator PC and each mobile robot.

The teleoperation of a multi-robot requires a throughput of 20.0 Mbps or higher in the communication path between each operator PC and each mobile robot. However, the theoretical value of IEEE802.11b/g used in RWSN is 54.0 Mbps, which is 
insufficient throughput for multi-robot operations. This study constructs a network using IEEE802.11ax, which has a theoretical value of approximately 1,200 Mbps.

\section{Topology of Multistage Relay Network}

Based on the process flow and requirements of multi-robot systems, this paper proposed a multistage relay network topology for the construction of multi-robot environments. The network topology shown in Fig. 5 is characterized by the fact that each node in the multistage relay network has an access point (AP) and multiple mobile robots can be connected to a single node. Therefore, in this topology, each node constructs a network, and multiple mobile robots can explore the network construction range exhaustively while changing the APs to be connected.

\section{Problems in Changing Communication Path}

In this multi-robot system, the mobile robot changes the search range while switching the connected nodes, and exhaustively explores within the network construction range (Fig. 6). However, when the mobile robot changes the node to be connected, the below problems occur.

- There is communication loss between the operator PC and the mobile robot while changing the node to be connected to.

- There communication quality is unknown in the communication path after changing the node to be connected.

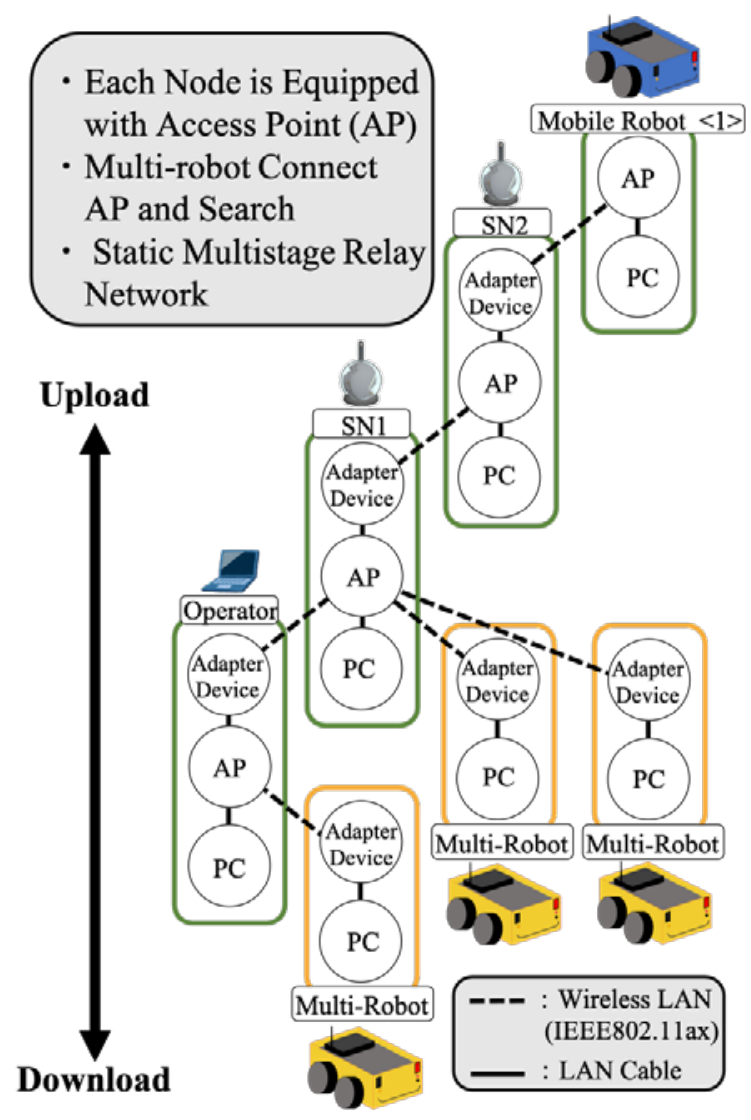

Fig. 5. Network Topology of Multi-robot System.

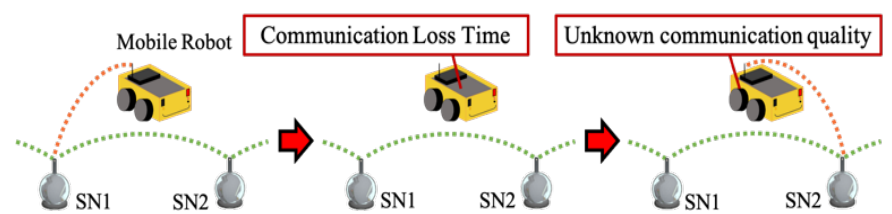

Fig. 6. Switching Nodes to Be Connected.

When the environment changes around the mobile robot during the communication loss time, the operator cannot receive sensor and camera information and cannot send operation command packets; consequently, it becomes difficult to respond to environmental changes. Moreover, when the connection change fails, the mobile robot becomes isolated. When the node to be connected is changed, the operator cannot determine whether the communication quality required for the teleoperation of the mobile robot can be maintained in the communication path after the node is changed. Therefore, there is a possibility of the mobile robot becoming isolated because of its inability to maintain communication connectivity after connecting to a new node. An isolated mobile robot is an obstacle to other mobile robots and affects their ability to explore the scene of disaster; they could also become a cause of secondary disasters because of their battery ignition. Therefore, this multi-robot system requires a method to change the node to which it is connected without losing the communication connectivity of the mobile robot. This paper proposes a communication path change method to maintain the communication connectivity of mobile robots.

\section{REQUIREMENTS FOR CHANGING COMMUNICATION PATH IN MULTI-ROBOT SySTEM}

Given the problems described in Section 2.2, the following specifications are required for the mobile robot to change the communication path in this multi-robot system.

1) The capability to send and receive packets between the operator PC and the mobile robot.

2) The capability to switch the communication path even when the operator does not know the exact location of the SN and the mobile robot.

3) Capable of obtaining the communication quality required for teleoperation of a mobile robot on the changed communication path, before switching the path.

During the exploration, it is possible to reduce the risk of isolation of the mobile robot by monitoring the changes in the environment and by being able to respond to them while the communication path was being changed. Therefore, as stated in specification (1), the system needs to be able to send and receive operation commands and camera/sensor information between the operator PC and the mobile robot during the communication path change.

After a disaster, a closed space would have an environment with multiple obstacles. In such cases, the SN and the mobile robot might not be able to see each other with a camera, or it might be difficult to measure the distance with a sensor because of the various disturbances. In such cases, there is a possibility of the mobile robot not being able to connect to the appropriate node and becoming isolated because of the lack of 
location information. Therefore, as stated in specification (2), the communication path is changed without depending on the location information.

Some studies have used the electric field strength as a method for switching the routing [22]. However, the teleoperation of mobile robots sends and receives packets; therefore, it is necessary to maintain the communication quality at the packet level. Therefore, specification (3) requires the maintenance of the throughput required for the teleoperation of the mobile robot in the changed communication path before changing the node. As described in Section 2.2, the throughput required for the teleoperation of the mobile robot in this system is $20.0 \mathrm{Mbps}$; therefore, in this paper, the throughput to be maintained in the communication path after the change was set to 20.0 Mbps.

\section{Method for Changing Communication Path WHILE MAINTAINING COMMUNICATION CONNECTIVITY FOR MOBILE ROBOTS}

This paper considers a method for changing the communication path of a mobile robot while maintaining communication connectivity in a multi-robot system using a multistage relay backbone network. This chapter proposes a method to obtain advance information about the communication quality of the changed communication path; this method will ensure that there is no communication loss time when the path is changed. In this method, apart from the main communication path, there is a sub-communication path that connects in advance to the next node. We also include a "judgment communication path" that monitors the communication quality. Section 4.1 describes a method to change the communication path without causing any communication loss; two communication paths were used here. Section 4.2 describes a method to determine in advance whether the communication quality required for the teleoperation of a mobile robot can be maintained in the communication path after changing the connecting nodes.

\section{A. Communication Path Changing Method using Sub- communication Path}

The proposed method used a mobile robot connected to two APs (IEEE802.11ax), as shown in Fig. 7. The steps for changing the communication path of a mobile robot using two communication paths are given below. Here, this paper assume that the node number is $n$ (Fig. 8).

1) The mobile robots connect to the $n$th and $n+1$ th nodes and teleoperate via the main communication path.

2) The communication quality of the judgment communication path is used to determine whether to switch between the main communication path and the subcommunication path.

3) The sub-communication path is changed to the main communication path.

4) The path that was originally the main communication path is changed to a sub-communication path. (The adapter device that was connected to the $n$th node is changed to the $n+2$ th node).

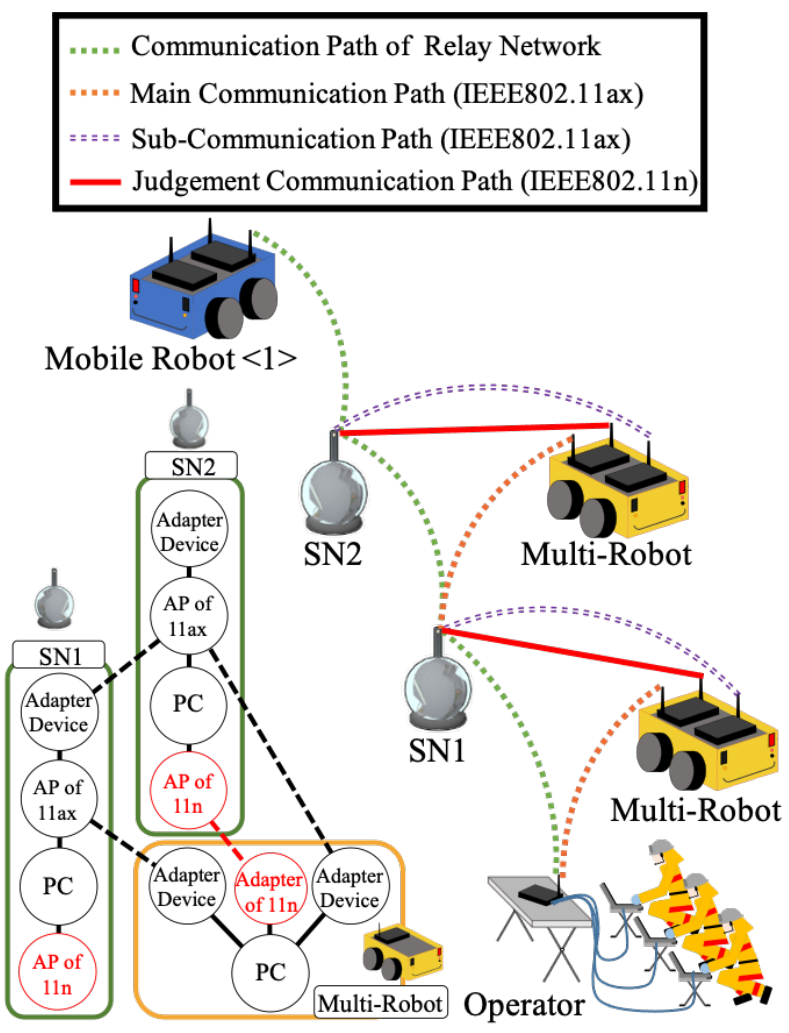

Fig. 7. Method for Changing Communication Path while Maintaining Communication Connectivity for Mobile Robots.

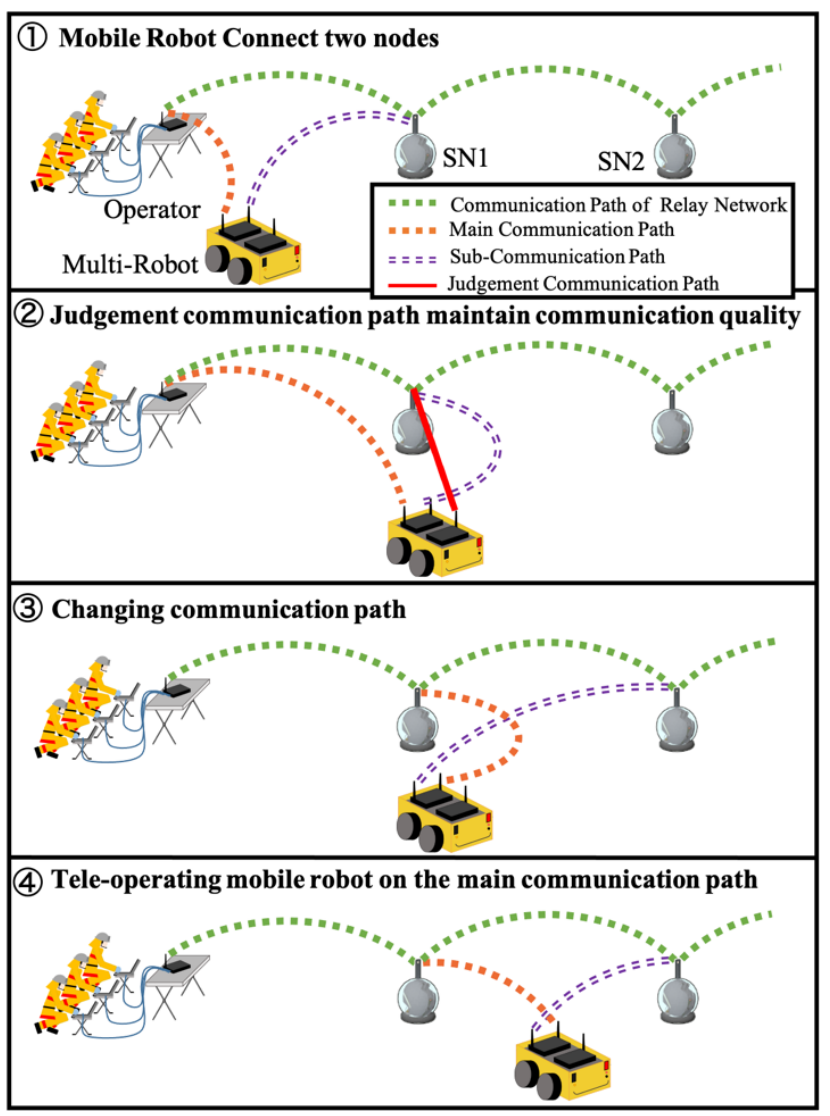

Fig. 8. Flow of Changing Communication Path. 
Two communication paths were used: one with a large number of relays and the other with a small number of relays. This paper consider the decrease in communication quality due to the increase in the number of relays, and use the communication path with a small number of relays as the main communication path for sending and receiving teleoperation packets. Then, the sub-communication path becomes the next main communication path, as shown in Fig. 8. The packets are sent and received to confirm that the communication has not been interrupted. The operator can communicate with the mobile robot on the path via the $n$th node when changing the sub-communication path to the main communication path. Then, when changing the main communication path to the subcommunication path, it is possible to communicate with the mobile robot via the $n+1$ th node. In this way, packets can be sent and received between the operator PC and the mobile robot while the communication route is being changed. Therefore, the mobile robots switch between the main communication path and the sub-communication path and can explore the disaster site without any communication loss even when there are changes in the communication path.

\section{B. Monitoring Communication Quality using Judgment Communication Path}

In the proposed method, in order to obtain in advance whether the communication path after changing the node to be connected can maintain the throughput required for teleoperation of the mobile robot, we construct a judgment communication path between the next node to be connected to and the mobile robot, as shown in Fig. 7. The judgment communication path is constructed by mounting an AP of IEEE802.11n, which is in the same frequency band as IEEE802.11ax, on the SN and an adapter device on the mobile robot. To obtain the throughput of this judgment communication path, the measurement packets are sent and received between the $\mathrm{SN}$ and the mobile robot $<1>$. This communication path is constructed in a separate network from the network of the multi-robot system, so that the throughput between the $\mathrm{SN}$ and the mobile robot can be measured without placing a load on the path used for teleoperation.

When the communication quality between an SN and a mobile robot of IEEE802.11n is maintained for the teleoperation of the mobile robot, the communication quality between an SN and a mobile robot of IEEE802.11ax can also be maintained for teleoperation of the mobile robot due to IEEE802.11n is in the same frequency band as IEEE802.11ax. This multi-robot system has also confirmed that the throughput between the operator PC and the $\mathrm{SN}$ is maintained required for the teleoperation of multiple mobile robots during the multistage relay network range is expanded. Therefore, if the communication quality required for the teleoperation of the mobile robot is maintained in the judgment communication path, it is assumed that the communication quality required for the teleoperation of the mobile robot is also maintained in the main communication path after switching the node to be connected. Therefore, in this method, the communication path is changed when the communication quality required for the teleoperation of the mobile robot can be maintained in the judgment communication path.

\section{EVALUATION OF COMMUNICATION QUALITY USING THROUGHPUT MEASUREMENT}

This chapter describes a method for measuring the communication quality characteristics when teleoperating a mobile robot. As described in Section 2, the teleoperation of a mobile robot sends and receives packets; therefore, it is necessary to evaluate the communication quality at the packet level. This paper evaluates the transmission speed in a TCP/IPcompliant communication path as the throughput at the packet level. The throughput [bps] specifies the transmission received per second by the PC. Therefore, the following experiments continue to send measurement packets from the mobile robot at a transmission speed of $25.0 \mathrm{Mbps}$ and confirm whether a throughput of $20.0 \mathrm{Mbps}$ or higher can be maintained at the receiving side. Therefore, the experiments in next chapter send measurement packets from the mobile robot side and confirm that the receiving side can maintain the throughput required for the teleoperation of the mobile robot.

\section{CHANGING COMMUNICATION PATH WHILE MAINTAINING COMMUNICATION CONNECTIVITY USING PROPOSED METHOD}

This experiment was conducted to confirm whether the proposed method can be used to change the communication path of a mobile robot while maintaining the communication connectivity. The experiment setting is shown in Fig. 9, and the equipment used is shown in Fig. 10. This experiment used Raspberry Pi 4 Model B as the PC, ASUS RT-AX3000 as the adapter device, GT-AX11000 as the AP of IEEE802.11ax, WN-AC433UA as the antenna of IEEE802.11n, and CAT8 LAN. The experiment was conducted in the following steps:

1) Deploying nodes equipped with AP of IEEE802.11n so that the distance between each node is $90 \mathrm{~m}$. (Constructing a 270m network with 3hop).

2) Move the mobile robot and measure the throughput in the judgment path of IEEE802.11n at $10 \mathrm{~m}$ intervals (Between each SN and the mobile robot).

3) Move the mobile robot and measure the throughput in the communication path of IEEE802.11ax at $10 \mathrm{~m}$ intervals. (Between operator PC and mobile robot via each node).

This experiment selected 10 throughput values measured at each location and shows the average of them. In the multistage relay network constructed in this experiment, the nodes are deployed so that the distance between each node is $90 \mathrm{~m}$ (270 $\mathrm{m}$ in total), and the network is capable of maintaining a throughput of more than $60.0 \mathrm{Mbps}$ between the operator PC and the mobile robot $<1>$. Fig. 11 shows the results of measuring the throughput on the judgment communication path (between SN and mobile robot). Fig. 11 shows that the throughput between SN1 and the mobile robot is more than 20.0 Mbps in the range of $60-130 \mathrm{~m}$. Therefore, the proposed method changes the sub-communication path to the main communication path in that section. The throughput between SN2 and the mobile robot is more than 20.0 Mbps in the range of $150-210 \mathrm{~m}$. Therefore, the proposed method changes the sub-communication path to the main communication path in that section. 


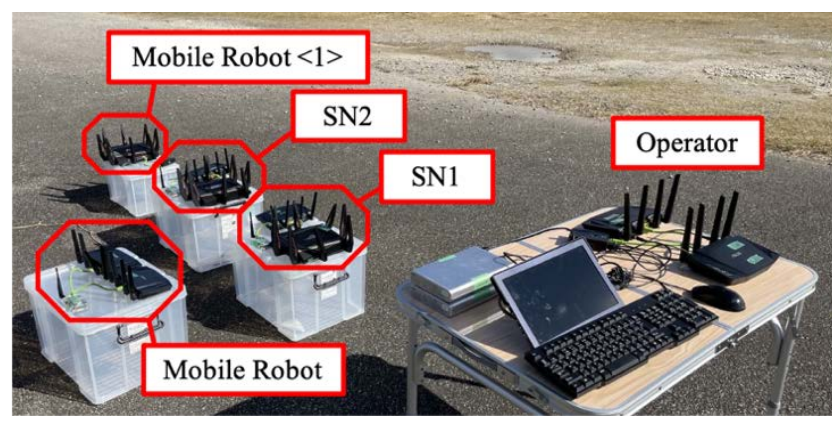

Fig. 9. Experimental Environment.

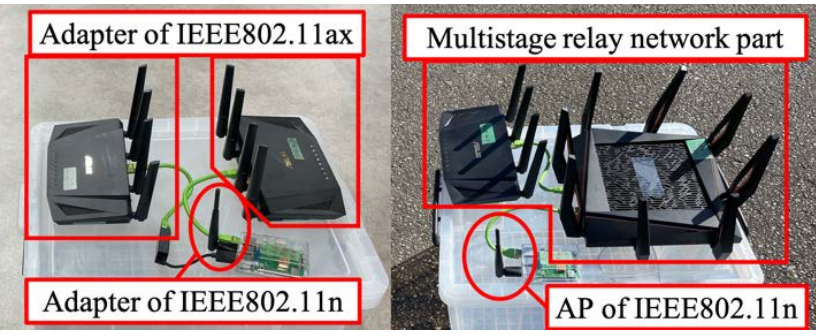

Fig. 10. Device of IEEE802.11n and IEEE802.11ax. (Left : SN, Right : Mobile Robot).

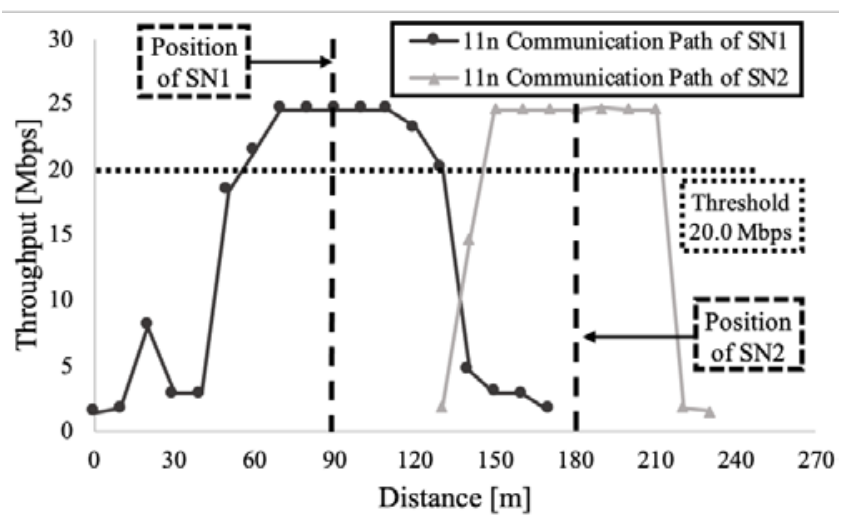

Fig. 11. Throughput of Judgment Communication Path (IEEE802.11n).

From the results shown in Fig. 11, we measured the throughput between the operator PC and the mobile robot so that the $60-130 \mathrm{~m}$ and $150-210 \mathrm{~m}$ sections overlap in this experiment, and confirmed whether the main communication path can be switched while maintaining the communication connectivity. The throughput in the communication path of IEEE802.11ax between the operator PC and the mobile robot via each node is shown in Fig. 12. As shown in Fig. 12, both communication paths have a throughput of more than $20 \mathrm{Mbps}$ in the $60-130 \mathrm{~m}$ section, so it was possible to switch the main communication path while maintaining the communication connectivity. Also, in the $150-210 \mathrm{~m}$ section, both communication paths are maintained at more than 20 Mbps, so it was possible to switch the main communication path while maintaining the communication connectivity. As a result, when the proposed method was used to teleoperate a mobile robot, the communication path could be switched while maintaining the communication connectivity. Therefore, this method is effective for changing the connected nodes in a multi-robot system using a multistage relay backbone network.

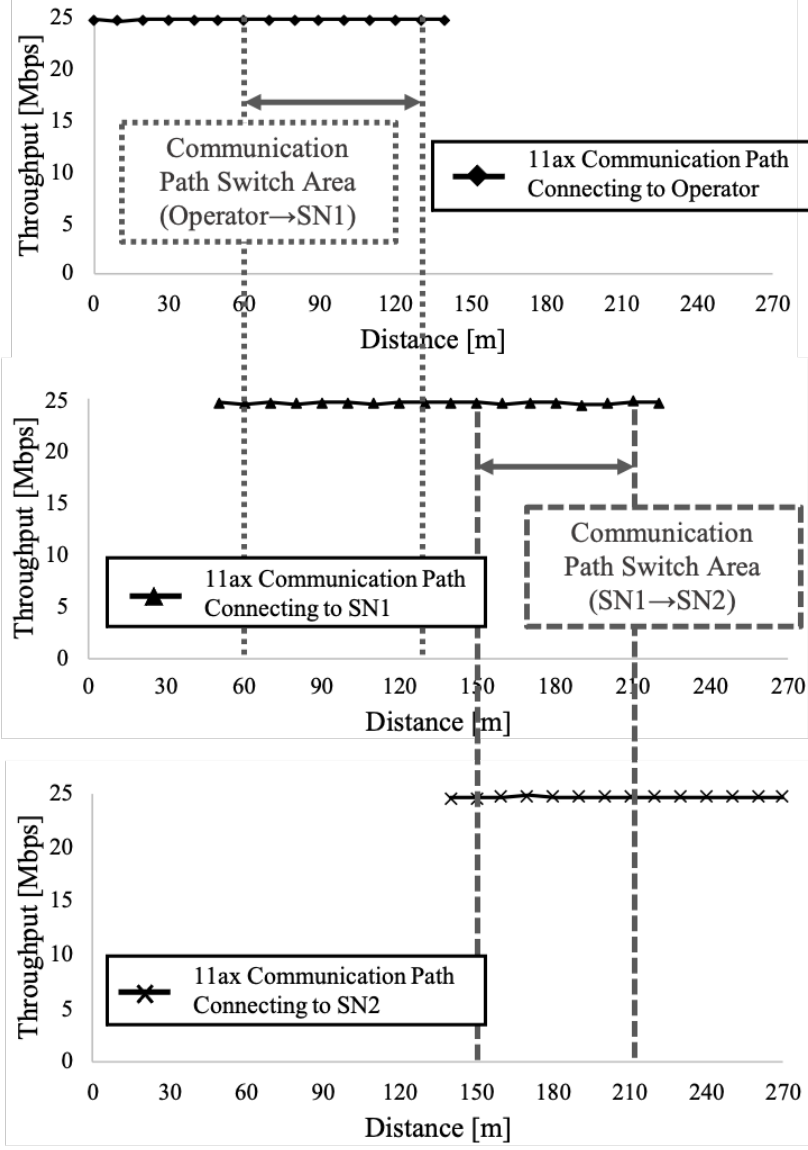

Fig. 12. Throughput between Operator and Mobile Robot. ( Top : Mobile Robot Connecting to Operator, Middle: Mobile Robot Connecting to SN1, Bottom: Mobile Robot Connecting to SN2).

\section{DISCUSSION}

In the judgment communication path of this experiment, the throughput between SN1 and the mobile robot is more than $20 \mathrm{Mbps}$ in the $60-130 \mathrm{~m}$ section, and the throughput between SN2 and the mobile robot is more than 20 Mbps in the 150-210 $\mathrm{m}$ section. The experimental results show that the IEEE802.11ax communication path can maintain a throughput of more than $20 \mathrm{Mbps}$ in those sections, so it is possible to change the main communication path after confirming the communication quality in the judgment communication path in advance. Therefore, proposal method can obtain the communication quality of the changed communication path in advance, without communication loss time when the path is changed. Additionally, switching between the subcommunication path and the main communication path does not depend on distance information, but on the throughput of the judgment communication path, which is effective even in environments with many obstacles.

\section{CONCLUSION}

In a multi-robot system using a multistage relay network, multiple mobile robots were connected to the constructed network, and the mobile robots changed the search range by switching between the nodes. However, a mobile robot can be isolated when changing the node because of the loss of 
communication or the poor quality of the communication in the new communication path. This paper proposed a method to change the communication path of a mobile robot using multiple paths so that communication connectivity is maintained.

The proposed method uses a sub-communication path, which connects in advance to the next node so that the communication path can be switched without any communication loss between the operator PC and the mobile robot. In addition, the proposed method constructs a judgment communication path between the nodes that constitute the subcommunication path; the main communication path and the sub-communication path are changed when the communication quality is maintained on the judgment communication path. Therefore, even when the sub-communication path is changed to the main communication path, the communication quality required for the teleoperation of the mobile robot can be maintained in the new main communication path. This study measured the throughput of the judgment communication path connected to each node and confirmed the section that can maintain the communication quality required for the teleoperation of the mobile robot. This experiment confirmed that the communication quality required for the teleoperation of the mobile robot could be maintained in the communication path of IEEE802.11ax in that section. The experiment results confirmed that it was possible to maintain the communication quality required for the teleoperation of the mobile robot in the new main communication path even when the main communication path was switched. The communication quality required for the teleoperation of the mobile robot was maintained in the judgment communication path. Therefore, the judgment communication path could monitor the communication quality of the changed path before changing the main communication path; this experiment proves the effectiveness of the proposed method.

In these experiments, the nodes to be connected to the mobile robot were changed manually. In the future, we will create a program that will automatically switch the communication path.

\section{REFERENCES}

[1] Yoshiaki Kawata, "The great Hanshin-Awaji earthquake disaster, damage, social response, and recovery," Journal of Natural Disaster Science, Vol. 17, No. 2, pp.1-12, 1995.

[2] L. Ernesto Dominguez-rios, Tomoko Izumi, Yoshio Nakatani, "A disaster management platform based on social network system oriented to the communities self-relief," IAENG International Journal of Computer Science, Vol. 42, No.1, pp.8-16, February 2015.

[3] Sabarish Chakkath, "Mobile robot in coal mine disaster surveillance," IOSR Jourbal of Engineering, Vol. 2, No. 10, pp. 77-82, 2012.

[4] Keiji Sakuradani, Keigo Koizumi, Kazuhiro Oda, Satoshi Tayama,” Development of a sloap disaster monitoring system for expressway operation and maintenance control,” Jounal of GeoEngineering, Vol. 13, No.4, pp.189-195, December 2018.

[5] F. Kurz, D. Rosenbaum, J. Leitloff, O. Meynberg, P. Reinartz, "A real time camera system for disaster nad traffic monitoring,". https://core.ac.uk/download/pdf/11146229.pdf.
[6] Jingxuan Sun, Boyang Li, Yifan Jiang, Chih-yung Wen, “A camerabased target detection and positioning UAV system for search and rescue (SAR) Purposes," Sensors 2016, Vol. 16, No. 11, 1778. https://doi.org/10.3390/s16111778.

[7] Masataka Fuchida, Shota Chikushi, Alessandro Moro, Atsushi Yamashita, Hajime Asama, "Arbitrary viewpoint visualization for teleoperation of disaster response robots," Journal of Advanced Simulation in Science and Engineering, Vol. 6, No. 1, pp.249-259, 2019.

[8] Hemanth Reddy A, Balla Kalyan, Ch. S. N. Murthy, "Mine Rescue Robot System - A Review," Procedia Earth and Planetary Science, Vol.11, pp. 457-462, 2015.

[9] Trupti B. Bhondve, Prof.R.Satyanarayan, Prof. Moresh Mukhedkar, "Mobile rescue robot for human body detection in rescue operation of disaster," International Journal of Advanced Research in Electrical, Electronics and Instrumentation Engineering, Vol.3, No.6, pp.98769882, June 2014.

[10] Zia Uddin, Mojaharul Islam, "Search and rescue system for alive human detection by semi-autonomous mobile rescue robot," International Conference on Innovations in Science, Engineering and Technology, Octorber 2016.

[11] Xuewen Rong, Rui Song, Xianming Song, Yibin Li, "Mechanism and explosion-proof design for a coal mine detection robot," Procedia Engineering, Vol. 15, pp.100-104, 2011.

[12] Tomoaki Yoshida, Keiji Nagatani, Satoshi Tadokoro, Takeshi Nishimura, Eiji Koyanagi, “ Improvements to the rescue robot Quince toward future indoor surveillance missions in the Fukushima Daiichi Nuclear Power Plant,” Field and Service Robotics, pp. 19-32, December 2013.

[13] Albert Ko, Henry Y. K. La, "Robot assisted emergency search and rescue system with a wireless sensor network," International Journal of Advanced Science and Technology, Vol. 3, pp.69-78, Febuary 2009.

[14] Andrew Wichmann, Burcu Demirelli Okkalioglu, Turgay Korkmaz, "The integration of mobile (tele) robotics and wireless sensor networks: A survey,” Computer Communications, Vol. 51, No.15, pp. 21-35, September 2014.

[15] Yasushi Hada, Osamu Takizawa, "Development of communication technology for search and rescue robots," Journal of the National Institute of Information and Communications Technology, Vol. 58, pp. 131-151, 2011.

[16] Carlos Marques, Joao Cristovao and Paulo Alvito, "A search and rescue robot with tele-operated tether docking system," Industrial Robot: An International Journal, Vol. 34, No. 4, pp. 332-338, 2007.

[17] Yuta Koike, Kei Sawai, Tsuyoshi Suzuki, "A study of routing path decision method using mobile robot based on distance between sensor nodes,” International Journal of Advanced Research in Artificial Intelligence, Vol. 3, No. 3, pp. 25-31, 2014.

[18] Kei Sawai, Ju Peng, Tsuyoshi Suzuki, “Throughput Measurement Method Using Command Packets for Mobile Robot Teleoperation Via a Wireless Sensor Network," (IJACSA) International Journal of Advanced Computer Science and Applications, Vol. 7, No. 4, pp 348- 354, 2016.

[19] Tsuyoshi Suzuki, Ryuji Sugizaki, Kuniaki Kawabata, Yasushi Hada, Yoshito Tobe, "Autonomous deployment and restoration of sensor network using mobile Robots," International Journal of Advanced Robotic Systems, Vol. 7, No. 2, pp. 105-114, 2010.

[20] M. Brett McMickell, Bill Goodwine, Luis Antonio Montestruque, "MICAbot: A robotic platform for large-scale distributed robotics," 2003 IEEE International Conference on Robotics and Automation, pp. 14-19, 2003.

[21] Peng Zeng, Jiahong He, Bingtuan Gao, "Reliable robot-flock-based monitoring system design via a mobile wireless sensor network," Sensor-Cloud Systems and Applications, Vol. 9, pp.47125-47135, 2021.

[22] Masayuki Tauchi, Tetsuo Ideguchi, Takashi Okuda, "Ad-hoc Routing Protocol Avoiding Route Breaks Based on AODV," Proceedings of the 38th Annual Hawaii International Conference on System Sciences, 2005. 